\title{
Predictors of Post-Partum Persistence of Glucose Intolerance and Its Association with Cardio-Metabolic Risk Factors in Gestational Diabetes Mellitus
}

\section{Sharmin Jahan, M Fariduddin, Nusrat-Sultana, Yasmin-Aktar, Mashfiqul-Hasan, Sandesh-Panthi, M Atiqur Rahman and M A Hasanat*}

Department of Endocrinology, Bangabandhu Sheikh Mujib Medical University (BSMMU), Shahbag, Dhaka, Bangladesh

\begin{abstract}
Objectives: To observe the impact of predictors [age and body mass index (BMI) of mother, family history of diabetes, parity, use of insulin in pregnancy, gestational age and glycemic status at diagnosis) on persistence of abnormal glucose tolerance (AGT) in GDM at 6-12 week post-partum and its relationship with cardio-metabolic risk factors.

Methods: This study encompassed 108 GDM subjects [age (year, $29.4 \pm 5.3)$; $\mathrm{BMI}\left(\mathrm{Kg} / \mathrm{m}^{2}, 26.4 \pm 4.97\right)$ mean \pm $\mathrm{SD}$ ] diagnosed by standard oral glucose tolerance test (OGTT) during index pregnancy. At 6-12 week post-partum, $75 \mathrm{~g}-2 \mathrm{hr}-\mathrm{OGTT}$ was done to know their glycemic status. Data for predictors and cardio-metabolic risk factors were obtained from history, clinical examination and personal records. Plasma glucose was measured by glucose-oxidase method on the same day of sampling.

Results: Age (yr, $31.3 \pm 5.8$ vs. $28.1 \pm 4.5, \mathrm{p}<0.001)$, BMI (kg/m², $27.9 \pm 4.7$ vs. $25.3 \pm 4.5, \mathrm{p}=0.004)$, FPG $(5.9$ \pm 0.21 vs. $5.3 \pm 0.08 \mathrm{mmol} / \mathrm{L}, \mathrm{p}=0.003)$ and 2 -hr-OGTT values $(10.7 \pm 0.40 \mathrm{vs} .9 .5 \pm 0.18 \mathrm{mmol} / \mathrm{L}, \mathrm{p}=0.006)$ (mean \pm SD) along with gestational age $<20$ weeks $(p<0.001)$ during detection of GDM at diagnosis, higher parity $(p<0.05)$ and insulin use during pregnancy $(p<0.001)$ were significantly higher in AGT than those of normal glucose tolerance (NGT). However, frequencies for AGT at post-partum considered on trimester of GDM detection $\left(1^{\text {st }}\right.$ trimester: $54.5 \%, 2^{\text {nd }}$ trimester: $46.6 \%$ and $3^{\text {rd }}$ trimester: $\left.33.3 \%\right)$ showed no statistical difference $(x 2=2.382, p=0.304)$. On stratification of FPG values during GDM detection, frequencies decreased for both AGT and NGT with increments of FPG at post-partum. Frequency of AGT was significantly higher than NGT with increment of FPG $(X 2=11.976$, $p=0.007$ ) which was also evident on dichotomization of FPG during GDM detection (at cut-off $5.1 \mathrm{mmol} / \mathrm{L}: \mathrm{p}=0.582$; at cut-off $6.1 \mathrm{mmol} / \mathrm{L}: \mathrm{p}=0.003$ and at cut-off $7.0 \mathrm{mmol} / \mathrm{L}: \mathrm{p}=0.001)$. Unlike overweight/obesity and family history of DM ( $p$ value $0.092 \& 0.138$ respectively), frequency of hypertension in AGT was higher than that of NGT ( $80 \%$ vs. $20 \%, p<0.002)$. FPG and 2-hr-OGTT value in $6-12$ week post-partum correlated in both AGT $(r=0.802, p<0.001)$ and NGT $(r=0.296, p=0.019)$, FPG at detection of GDM with post-partum FPG of AGT $(r=0.594, p<0.001$ postpartum 2-hr-OGTT glucose in AGT $(r=0.499, p<0.001)$. AGT correlated inversely with age of gestation at GDM detection $(r=-0.196, p=0.042)$ whereas positively with hypertension $(r=0.304, p<0.001)$ and use of insulin during pregnancy $(r=0.421, p<0.001)$. Multiple regression revealed that age of the subjects $(p=0.009)$ and use of insulin during pregnancy $(p=0.035)$ were independent predictors of $A G T$ at post-partum.
\end{abstract}

Conclusion: It is concluded that advanced age, higher BMI, magnitude of both FPG and 02-hr glucose value of OGTT, earlier gestational age at diagnosis of GDM and use of insulin during pregnancy are important predictors for AGT during post-partum period and hypertension as a cardio metabolic risk factor was strongly associated with AGT.

Keywords: GDM; Post-partum glucose intolerance and its predictors; Cardio-metabolic risk factors

\section{Introduction}

Prevalence of gestational diabetes mellitus (GDM) varies among different population [1]. Recently, we observed an alarming frequency under a pilot project in a tertiary care based hospital in Bangladesh [2]. Usually soon after giving birth, $90 \%$ to $95 \%$ women with GDM are diabetes-free by $75 \mathrm{~g}$-oral glucose tolerance test (OGTT). However, by $6-12$ weeks post-partum, $4 \%$ to $9 \%$ are diagnosed with type2 DM; more than $20 \%$ have impaired glucose tolerance (IGT) or impaired fasting glucose (IFG) or both [3]. It has been observed that one third to one half of women with history of GDM develop type-2 $\mathrm{DM}$ within 3 to 5 years and the figure may reach $70 \%$ over 10 years having a cumulative incidence of $2.6 \%$ to $70 \%$ [4]. The effect of GDM extends beyond pregnancy for both the mother and the child. Women with a history of GDM are not only at a substantially higher risk of type $2 \mathrm{DM}$, but also vascular dysfunction, cardiovascular disease, metabolic syndrome and its components including hypertension [5]. It is generally accepted that race, age, parity, family history of DM, pre-pregnancy weight, post-partum obesity and weight gain are risk factors for developing type $2 \mathrm{DM}$. Other associated risk factors include smoking, physical inactivity, diet and drugs that adversely affect glucose metabolism [4]. Ethnicity is one of the key determinants of cumulative risk for abnormal glucose tolerance (AGT) at post-partum. Thus, the risk varies from nation to nation [6]. GDM is said to predict

${ }^{*}$ Corresponding author: Prof. M A Hasanat, Professor of Endocrinology, Department of Endocrinology,D Block, Room No. 1620, BSSMU, Shahbag, Dhaka Bangladesh, Tel: 01711670735; E-mail: hasanatdr@yahoo.com

Received August 18, 2015; Accepted September 23, 2015; Published September 28, 2015

Citation: Jahan S, Fariduddin M, Sultana N, Aktar Y, Hasan M, et al. (2015) Predictors of Post-Partum Persistence of Glucose Intolerance and Its Association with Cardio-Metabolic Risk Factors in Gestational Diabetes Mellitus. J Diabetes Metab 6: 609. doi:10.4172/2155-6156.1000609

Copyright: $\odot 2015$ Jahan S, et al. This is an open-access article distributed under the terms of the Creative Commons Attribution License, which permits unrestricted use, distribution, and reproduction in any medium, provided the original author and source are credited. 
Citation: Jahan S, Fariduddin M, Sultana N, Aktar Y, Hasan M, et al. (2015) Predictors of Post-Partum Persistence of Glucose Intolerance and Its Association with Cardio-Metabolic Risk Factors in Gestational Diabetes Mellitus. J Diabetes Metab 6: 609. doi:10.4172/2155-6156.1000609

Page 2 of 5

the future development of type $2 \mathrm{DM}$ which is commonly associated with various components of metabolic syndrome like being overweight or obese, presence of positive family history of diabetes, hypertension, dyslipidaemia, mainly characterized by elevated TG and low HDL [7]. As with lipids, continuing investigation of the metabolic syndrome and CVD risk factors is justified after GDM, to determine the likelihood of CVD events and means of their prevention. If a woman is found to be diabetic by above criteria then she should be referred for diabetic management, but if pre-diabetic then $\mathrm{HbA}_{1} \mathrm{c}$ test is recommended annually; if within normal limit, then every 3 years.

This study was intended to observe the impact of the above mentioned predictors over persistence of AGT at post-partum and its association with cardio-metabolic risk factors.

\section{Methods}

\section{Study subjects}

This study encompassed 108 patients diagnosed as GDM on the basis of 75/100-gm-OGTT during their index pregnancy. Informed written consent for participation in the study was taken from each of them. Those having history or clinical manifestation of diabetes or use of anti-diabetic drugs before pregnancy, history or clinical manifestation of cardiovascular disease or other co-morbidities (judged on clinical assessment) or having a BMI $\geq 40 \mathrm{~kg} / \mathrm{m}^{2}$ were excluded from the study. Characters of the study subjects are shown in Table 1.

\section{Study design}

It was a cross-sectional study carried out at the GDM Clinic, Department of Endocrinology, Bangabandhu Sheikh Mujib Medical University (BSMMU) from December, 2011 to June, 2013. Subjects were enrolled consecutively on the basis of the OGTT results during index pregnancy. All the subjects had been on unrestricted carbohydrate diet for 3 days, and came to GDM Clinic after an overnight fast (at least $8 \mathrm{hr}$ but not more than $14 \mathrm{hr}$ ) to undergo a formal 75-g 2-h OGTT at 6-12 weeks post-partum. Data on demographic characteristics, parity, family history of diabetes, OGTT report and insulin use during pregnancy etc. were collected in prescribed sheet. Clinical evaluation including estimation of height, weight, BMI $\left(\mathrm{kg} / \mathrm{m}^{2}\right)$ and $\mathrm{BP}(\mathrm{mmHg})$ was done for each subject by the same investigator. Prior to commencement of this study the research protocol was approved by Institutional Review Board (IRB).

\section{Analytic methods}

Plasma glucose was assayed immediately by glucose-oxidase method (Dade Behring machine, Germany). The result was deducted from computerized calculation utilizing standard curve derived from known concentrations used by the system. Samples of different subjects were run on different days in different assay runs (63 runs). A fixed known concentration for low level $(5.2 \mathrm{mmol} / \mathrm{l})$ as well as high level $(16.1 \mathrm{mmol} / \mathrm{l})$ was used in every assay run. Inter-assay co-efficient variance $(\mathrm{CV})$ for low level was $5.78 \%$, and for high level 5.59\%.

\section{Statistical analysis}

All data were analyzed by the use of SPSS program (version 22.0) and expressed as mean \pm SEM or in frequency or percentage unless mentioned otherwise. Test for cardio-metabolic risk factors between groups with and without intolerance was done by Student's unpaired t-test or Chi-square test as applicable and multiple regressions was done to predict over the factors capable of indicating the possibility of persistence of glucose intolerance during postpartum period.
Correlation between glucose level and age of subject, gestational age at diagnosis of GDM, BMI were analyzed by Pearson's and/or Spearman correlation. $P$ values $\leq 0.05$ were considered statistically significant.

\section{Results}

\section{Post-partum Glycemic status- its predictors and association} with cardio-metabolic risk

Age (year, $31.3 \pm 5.8$ vs. $28.1 \pm 4.5, \mathrm{p}<0.001)$ and $\mathrm{BMI}\left(\mathrm{Kg} / \mathrm{m}^{2}\right.$, $27.9 \pm 4.7$ vs. $25.3 \pm 4.5, \mathrm{p}=0.004$ ) (AGT vs. NGT, mean $\pm \mathrm{SD}$ ) were statistically higher in AGT group. Similarly, FPG (5.9 \pm 0.21 vs. $5.3 \pm$ $0.08 \mathrm{mmol} / \mathrm{L}, \mathrm{p}=0.003$ ) as well as 02 -hr-OGTT value during detection of GDM (10.7 \pm 0.40 vs. $9.5 \pm 0.18 \mathrm{mmol} / \mathrm{L}, \mathrm{p}=0.006)$ (AGT vs. NGT, mean $\pm \mathrm{SD}$ ) were significantly higher in those with AGT. Gestational age $<20$ week at detection of GDM $(\mathrm{p}<0.001)$, higher parity $(\mathrm{p}<0.05)$ as well as insulin use during pregnancy $(\mathrm{p}<0.001)$ were also significantly higher in the AGT group (Table 2). As shown in Table 3, increments

\begin{tabular}{|c|c|}
\hline Character & Value (\%) \\
\hline Number & 108 \\
\hline Age (mean $\pm S D$, year) & $29.4 \pm 5.3$ \\
\hline $\mathrm{BMI}\left(\mathrm{Kg} / \mathrm{m}^{2}\right.$, mean $\left.\pm \mathrm{SD}\right)$ & $26.4 \pm 4.7$ \\
\hline \multicolumn{2}{|l|}{ Parity } \\
\hline 0 & Nil \\
\hline 1 & $34(31.5)$ \\
\hline 2 & $36(33.3)$ \\
\hline$\geq 3$ & $38(35.2)$ \\
\hline Family H/O DM & $64(59.3)$ \\
\hline \multicolumn{2}{|l|}{ Gestational age at detection of GDM } \\
\hline$<20$ week & $34(31.5)$ \\
\hline$\geq 20$ week & $74(68.5)$ \\
\hline Insulin use during pregnancy & $23(21.3)$ \\
\hline $\begin{array}{l}\text { (Within parenthesis are percentages } \\
\text { BMI= Body Mass Index } \\
\text { DM= Diabetes Mellitus } \\
\text { GDM= Gestational Diabetes Mellitus }\end{array}$ & \\
\hline
\end{tabular}

Table 1: Characteristics of studied subjects.

\begin{tabular}{|c|c|c|c|}
\hline Variable & AGT $(n=46)$ & NGT $(n=62)$ & $\mathbf{p}$ \\
\hline Age(Mean $\pm S D$, year) & $31.3 \pm 5.8$ & $28.1 \pm 4.5$ & $<0.001$ \\
\hline $\mathrm{BMI}\left(\right.$ Mean $\left.\pm \mathrm{SD}, \mathrm{Kg} / \mathrm{m}^{2}\right)$ & $27.9 \pm 4.7$ & $25.3 \pm 4.5$ & 0.004 \\
\hline \multicolumn{4}{|l|}{ Parity } \\
\hline 0 & 00 & 00 & \multirow{4}{*}{0.0517} \\
\hline 1 & $13(38.2)$ & $21(61.8)$ & \\
\hline 2 & $14(38.9)$ & $22(61.1)$ & \\
\hline$\geq 3$ & $19(50.0)$ & $19(50.0)$ & \\
\hline \multicolumn{4}{|l|}{ Family H/O DM } \\
\hline Present & $31(48.4)$ & $33(51.6)$ & \multirow{2}{*}{0.138} \\
\hline Absent & $15(84.1)$ & $29(65.9)$ & \\
\hline \multicolumn{4}{|l|}{ Insulin during pregnancy } \\
\hline Yes & $19(82.6)$ & $04(17.4)$ & \multirow[b]{2}{*}{$<0.001$} \\
\hline No & $27(36.5)$ & $58(68.2)$ & \\
\hline \multicolumn{4}{|l|}{ Gestational age at diagnosis of GDM: } \\
\hline$<20$ week & $19(55.9)$ & $15(44.1)$ & \multirow{2}{*}{$<0.001$} \\
\hline$\geq 20$ week & $27(36.5)$ & $47(63.5)$ & \\
\hline FPG of OGTT of pregnancy (Mean \pm SEM) & $5.9 \pm 0.21$ & $5.3 \pm 0.08$ & 0.003 \\
\hline Post 2Hr-OGTT of pregnancy (Mean \pm SEM) & $10.7 \pm 0.40$ & $9.5 \pm 0.18$ & 0.006 \\
\hline \multicolumn{4}{|c|}{$\begin{array}{l}\text { (Values within parenthesis are percentage over row total) } \\
\text { AGT= Abnormal Glucose Tolerance; NGT= Normal Glucose Tolerance } \\
\text { BMI= Body Mass Index }\end{array}$} \\
\hline
\end{tabular}

Table 2: Comparison of risk variables between AGT and NGT. 
Citation: Jahan S, Fariduddin M, Sultana N, Aktar Y, Hasan M, et al. (2015) Predictors of Post-Partum Persistence of Glucose Intolerance and Its Association with Cardio-Metabolic Risk Factors in Gestational Diabetes Mellitus. J Diabetes Metab 6: 609. doi:10.4172/2155-6156.1000609

Page 3 of 5

\begin{tabular}{|c|c|c|c|}
\hline \multirow{2}{*}{$\begin{array}{l}\text { FPG cut-offs (mmol/L) } \\
\text { during detection of GDM }\end{array}$} & \multicolumn{2}{|c|}{ Frequencies } & \multirow{2}{*}{$x 2 \& p$} \\
\hline & AGT & NGT & \\
\hline$<6.1$ & $30(65.2)$ & $55(88.7)$ & \multirow{5}{*}{$\begin{array}{c}11.979 \\
0.007\end{array}$} \\
\hline $6.1-6.5$ & 05 (10.9) & $05(8.1)$ & \\
\hline $6.51-7.0$ & $02(4.3)$ & $01(1.6)$ & \\
\hline$>7.0$ & 09 (19.6) & $01(1.6)$ & \\
\hline Total & 46 & 62 & \\
\hline
\end{tabular}

Table 3: Frequency of abnormal glucose tolerance at various cut-off values of fasting glucose at detection of GDM.

\begin{tabular}{|c|c|c|c|}
\hline Dichotomized FPG cut-off value & AGT $(n=46)$ & NGT $(n=62)$ & $\mathbf{p}$ \\
\hline \multicolumn{4}{|l|}{ Cut-off at $5.1 \mathrm{mmol} / \mathrm{L}$} \\
\hline$<5.1$ & $14(38.9)$ & $22(61.1)$ & \multirow{2}{*}{0.582} \\
\hline$\geq 5.1$ & $32(44.4)$ & $40(55.6)$ & \\
\hline \multicolumn{4}{|l|}{ Cut-off at $6.1 \mathrm{mmol} / \mathrm{L}$} \\
\hline$<6.1$ & $30(35.3)$ & $55(64.7)$ & \multirow{2}{*}{0.003} \\
\hline$\geq 6.1$ & $16(69.6)$ & $07(30.4)$ & \\
\hline \multicolumn{4}{|l|}{ Cut-off at $7.0 \mathrm{mmol} / \mathrm{L}$} \\
\hline$<7.0$ & $37(37.8)$ & $61(62.2)$ & \multirow{2}{*}{0.001} \\
\hline$\geq 7.0$ & $09(90.0)$ & $01(10.0)$ & \\
\hline
\end{tabular}

(Values within parenthesis are percentage over row total); Groups are not mutually exclusive

FPG=Fasting Plasma Glucose

GDM=Gestational Diabetes Mellitus; AGT=Abnormal Glucose Tolerance; NGT=Normal Glucose Tolerance

Table 4: Frequency of AGT in post-partum period under various dichotomized cutoff values of FPG at detection of GDM.

\begin{tabular}{|c|c|c|c|c|}
\hline \multirow{2}{*}{$\begin{array}{l}\text { Trimester at } \\
\text { detection of GDM }\end{array}$} & \multicolumn{2}{|c|}{ Frequencies } & \multirow{2}{*}{ Total } & \multirow{2}{*}{$x^{2} \& p$} \\
\hline & AGT & NGT & & \\
\hline $1^{\text {st }}$ & $06(54.5)$ & $05(45.5)$ & 11 & \multirow{4}{*}{$\begin{array}{l}2.382 \\
0.304\end{array}$} \\
\hline $2^{\text {nd }}$ & $27(46.6)$ & $31(53.4)$ & 58 & \\
\hline $3 r d$ & $13(33.3)$ & $26(66.7)$ & 39 & \\
\hline Total & 46 & 62 & 108 & \\
\hline
\end{tabular}

Table 5: Frequency of AGT in post-partum in subjects detected as GDM at various trimester of pregnancy.

of FPG were negatively associated with frequencies of both AGT and NGT groups; but frequency of AGT relatively more with increment of FPG ( $\chi 2=11.976, p=0.007)$. On dichotomization of FPG at GDM diagnosis (Table 4), frequency of AGT gradually increased with increment of FPG (for cut-off $5.1 \mathrm{mmol} / \mathrm{L}: \mathrm{p}=0.582$; cut-off 6.1 $\mathrm{mmol} / \mathrm{L}: \mathrm{p}=0.003$ and cut-off at $7.0 \mathrm{mmol} / \mathrm{L}: \mathrm{p}=0.001)$. However, of the total 108 GDM subjects, 11 performed OGTT in $1^{\text {st }}$ trimester which was 58 in $2^{\text {nd }}$ and 39 in $3^{\text {rd }}$ trimester (Table 5). There was no statistical difference $(\chi 2=2.382, \mathrm{p}=0.304)$ among the trimesters (AGT vs. NGT: $54.5 \%$ vs. $45.5 \%$; $46.6 \%$ vs. $53.4 \%$ and $33.3 \%$ vs. $66.7 \%$ respectively) for persistence of glucose intolerance at post-partum. Figure 1 shows that the frequency of being overweight/ obese and having family history of DM were not statistically different ( $\mathrm{p}$ value $0.092 \& 0.138$ respectively) unlike hypertension (AGT vs. NGT; $80 \%$ vs. $20 \%$, p<0.002) between AGT and NGT.

\section{Correlation and logistic regressions}

FPG and 02-hr-OGTT in 6-12 week post-partum significantly correlated in both AGT $(r=0.802, p<0.001)$ and NGT $(r=0.297$, $\mathrm{p}<0.019)$ groups. FPG at detection of GDM also strongly correlated with FPG at postpartum AGT $(\mathrm{r}=0.594, \mathrm{p}<0.001)$ but weakly with NGT $(\mathrm{r}=0.221, \mathrm{p}<0.085)$. Similarly, 02-hr-OGTT glucose at GDM detection also strongly correlated with post-partum 02-hr-OGTT glucose in AGT $(r=0.499, \mathrm{p}<0.001)$ but not in NGT $(\mathrm{r}=0.104, \mathrm{p}=0.402)$ (Table 6). As shown in Table 7, age of gestation at GDM detection inversely correlated with AGT $(\mathrm{r}=-0.196, \mathrm{p}=0.042)$ whereas hypertension $(\mathrm{r}=0.304, \mathrm{p}<0.001)$ and use of insulin during pregnancy $(\mathrm{r}=0.421$, $\mathrm{p}<0.001)$ were positively correlated with AGT; but neither parity nor family history of DM showed any significant correlation ( $p=N S$ ). Multiple regression analysis for persistence of glucose intolerance during 6-12 week post-partum period (Table 8) revealed that age of the subjects $(\mathrm{p}=0.009)$ and use of insulin during pregnancy $(\mathrm{p}=0.035)$ were independent predictors for persistence of glucose intolerance among the probable risk factors.

\section{Discussion}

In the present study, we analyzed over the impact of various risk variables predicting the persistence of glucose intolerance at 6-12 week post-partum. Some of these factors were related to the patient's clinical history and physical examination, while others emanated from
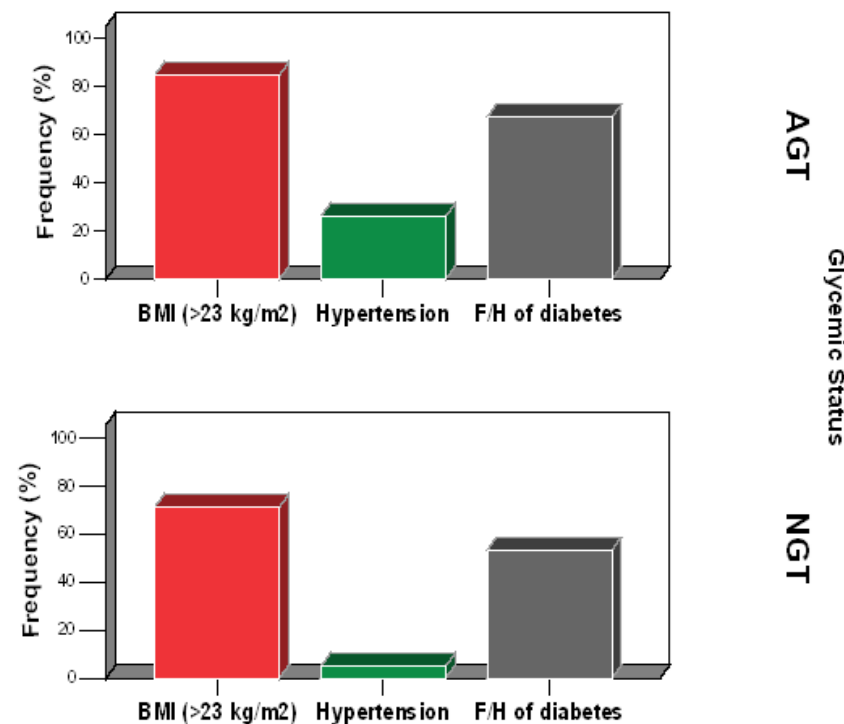

$\frac{2}{9}$

Figure 1: Frequency of overweight, hypertension and family history of diabetes. Percentages are shown over grand total in the diagram. NGT: normal glucose tolerance AGT: abnormal glucose tolerance BMI: body mass index.

\begin{tabular}{|l|c|c|c|c|}
\hline \multirow{2}{*}{ Variables } & \multicolumn{2}{|c|}{ AGT } & \multicolumn{2}{|c|}{ NGT } \\
\cline { 2 - 5 } & $\mathbf{r}$ & $\mathbf{p}$ & $\mathbf{r}$ & $\mathbf{p}$ \\
\hline Age vs. FPG & -0.122 & 0.420 & 0.113 & 0.382 \\
\hline Age vs. 2hr-OGTT value & 0.026 & 0.865 & -0.048 & 0.709 \\
\hline Age vs. BMI & 0.123 & 0.415 & 0.102 & 0.432 \\
\hline FPG vs. BMI & 0.125 & 0.408 & 0.186 & 0.148 \\
\hline $\begin{array}{l}\text { FPG in postpartum vs. 2 hr-OGTT } \\
\text { postpartum }\end{array}$ & 0.802 & $<0.001$ & 0.297 & 0.019 \\
\hline $\begin{array}{l}\text { FPG in Pregnancy vs. FPG } \\
\text { postpartum }\end{array}$ & 0.594 & $<0.001$ & 0.221 & 0.085 \\
\hline $\begin{array}{l}\text { 2 Hr. glucose in Pregnancy vs. 2 Hr. } \\
\text { glucose postpartum }\end{array}$ & 0.499 & $<0.001$ & 0.104 & 0.402 \\
\hline
\end{tabular}

AGT = Abnormal Glucose Tolerance; NGT= Normal Glucose Tolerance; FPG= Fasting Plasma Glucose; OGTT= Oral Glucose Tolerance Test; BMI= Body Mass Index

Table 6: Correlations of risk variables with glycemic status. 


\begin{tabular}{|l|c|c|}
\hline Variables & $\mathbf{r}$ & $\mathbf{p}$ \\
\hline Age of gestation at GDM detection & -0.196 & 0.042 \\
\hline Parity & 0.099 & 0.906 \\
\hline Hypertension & 0.304 & $<0.001$ \\
\hline Family history of diabetes & 0.143 & 0.141 \\
\hline Use of insulin during pregnancy & 0.421 & $<0.001$ \\
\hline GDM= Gestational Diabetes Mellitus & & \\
\hline
\end{tabular}

Table 7: Correlations with persistence of Glucose intolerance (by Spearman correlation).

\begin{tabular}{|l|c|c|c|}
\hline Variables & $\boldsymbol{\beta}$ & SE & $\mathbf{p}$ \\
\hline Constant & - & 0.475 & 0.037 \\
\hline Age & 0.260 & 0.009 & 0.009 \\
\hline BMI & 0.103 & 0.010 & 0.287 \\
\hline Gestational age at GDM detection & -0.066 & 0.006 & 0.458 \\
\hline Parity & -0.045 & 0.038 & 0.637 \\
\hline F/H of DM & -0.010 & 0.095 & 0.920 \\
\hline Use of insulin & 0.226 & 0.128 & 0.035 \\
\hline FPG during pregnancy & 0.112 & 0.056 & 0.358 \\
\hline Post OGTT 2hr glucose in pregnancy & 0.102 & 0.028 & 0.394 \\
\hline
\end{tabular}

$\mathrm{BMI}=$ Body Mass Index; DM=Diabetes Mellitus; FPG=Fasting Plasma Glucose; OGTT=Oral Glucose Tolerance Test

Table 8: Multiple regressions for persistence of glucose intolerance.

relevant biochemical parameter (e.g. OGTT report of pregnancy). It was clearly observed that advancing age, higher BMI, multiparity, earlier gestational age ( $<20$ week) at GDM detection, insulin use during pregnancy all were significantly higher in the group of AGT. Out of the CVD risk factors investigated, only hypertension but not obesity/ overweight or family history was found to be significantly related. When considered against trimester specific detection of GDM, there was no significant difference in the persistence of glucose intolerance during post-partum though frequencies were higher in the $1^{\text {st }}$ and $2^{\text {nd }}$ trimester.

Positive family history of DM reached near significance level as predictor for persistence of glucose intolerance at 6-12 week postpartum. It is probable that if large number of subjects were incorporated in the study, this might have reached the level of significance. However, biochemical parameter like higher mean FPG as well as $2 \mathrm{hr}$-OGTT value at detection of GDM were both significantly higher in AGT group than NGT group. As observed higher the FPG during GDM detection, lower was the frequencies of both AGT and NGT; but frequencies of AGT were relatively more with higher FPG categories. It was noted that the post-partum FPG level was significantly higher in all subgroups of FPG level of pregnancy in the AGT group. Unlike NGT group, the post-partum FPG in AGT group reflected to and correlated with some extent to the FPG and 2hr-OGTT observed during antepartum OGTT. Apropos with these findings, on dichotomization of FPG at GDM diagnosis, frequency of AGT gradually increased with higher cut-off values. On the contrary, FPG during ante partum OGTT would not focus on the possibility of having NGT at post-partum. So, a formal, complete $2 \mathrm{hr}-75 \mathrm{~g}$-OGTT is required for both GDM screening in antenatal period and to see persistence of intolerance 6-12 week post-partum. Similarly, when $2 \mathrm{hr}$-OGTT value at $6-12$ week post-partum were considered on the basis of antenatal FPG level, it was observed that AGT group had significantly higher 2 hr-OGTT glucose level than the NGT group. 2 hr-OGTT value during detection of GDM was also stratified into groups to assess the impact over $2 \mathrm{hr}$-OGTT value at 6-12 week post-partum; it was statistically significant in the AGT group than NGT in all subgroups. Lam et al. (1991) analyzed over the clinical variables and showed that a high fasting glucose during pregnancy and at the first post-natal visit, high ante partum 2-hr blood glucose, insulin requirement during pregnancy and high postpartum $2 \mathrm{hr}$ glucose all were predictive of AGT postpartum [8]. On the other hand, macrosomia, gestational age at diagnosis and a family history of DM were not predictive of persistence of glucose intolerance. Multiparity, maternal age, BMI were of marginal significance only. Other studies also noted such factors as predictors of persistence of AGT [9-11]. Kim et al. described the degree of severity of glucose intolerance in pregnancy as the most important independent predictor of persistence of AGT [12].

A substantial number of women with prior GDM share many characteristics with subjects that have the metabolic syndrome (e.g., glucose intolerance, insulin resistance, central obesity, elevated triglyceride and low HDL cholesterol) and inflammatory markers (e.g., high sensitivity C-reactive protein and interleukin 6). It has been suggested that chronic states of insulin resistance may produce chronic inflammation, adversely affecting vascular reactivity and atherogenesis, and may set up future hypertension and ischemic vascular disease in these women [7]. In the backdrop of the aforementioned information, we tried to assess the association of persistence of abnormal glucose tolerance at 6-12 week post-partum with certain CVD risk factors. Out of these only hypertension was found to be statistically significant in the persister group whereas, being overweight or obese or presence of positive family history of diabetes were not found to be statistically significant. Kitzmiller et al. investigated 481 GDM women in Denmark over 4-23 year and reported that $68 \%$ had impaired glucose regulation, $59 \%$ elevated fasting serum insulin, $54 \%$ central obesity, $28 \%$ hypertension and $35 \%$ dyslipidaemia, mainly characterized by elevated TG and low HDL [13]. Total plasma homocysteine level was a risk factor for the development of diabetes after GDM. As with lipids, continuing investigation of the metabolic syndrome and CVD risk factors is justified after GDM, to determine the likelihood of CVD events and means of their prevention.

While conducting this study we confronted multiple obstacles, most notably poor adherence to post-partum testing. Though there was a long list of subjects in our recruitment diary, only a few reported for post-partum testing despite repeated reminder over the telephone. A convenient window period of 6-12 week post-delivery was offered for the benefit of the mothers, yet many failed to respond. Probable factors leading to non-adherence to testing were lack of awareness, superstition, and difficulty in time management with a small baby. Other studies also observed similar poor rates of adherence to postpartum testing $[14,15]$.

\section{Conclusions}

This study showed that the rate of persistence of glucose intolerance at 6-12 week post-partum was quite high among post-pregnant population of Bangladesh. Out of the predictors of persistence of post-partum AGT, advancing age, higher BMI, multiparity, earlier gestational age at GDM detection ( $<20$ week), insulin use during pregnancy, higher FPG as well as 2hr-OGTT value at detection of GDM all were important statistically. Of the CVD risk factors hypertension was found to be significantly associated with persistence of glucose intolerance. Continued follow-up over time is required, as because the NGT might convert to AGT thereby increasing the prevalence of persistence. Management of diabetes risk in these mothers should be coupled with appropriate family planning and with efforts to detect and minimize the development of obesity in her children in order to arrest the vicious cycle of trans-generational diabetes. 
Citation: Jahan S, Fariduddin M, Sultana N, Aktar Y, Hasan M, et al. (2015) Predictors of Post-Partum Persistence of Glucose Intolerance and Its Association with Cardio-Metabolic Risk Factors in Gestational Diabetes Mellitus. J Diabetes Metab 6: 609. doi:10.4172/2155-6156.1000609

Page 5 of 5

\section{Acknowledgements}

The authors gratefully acknowledge the scientific colleagues of the department for their generous and moral support to the project. Supporting staffs of the department and patients participating in the project are also thanked and appreciated. Novo Nordisk Pharma Bangladesh is acknowledged for their financial help.

\section{References}

1. Reinblatt SL, Morin L, Meltzer SJ (2006) The importance of a postpartum 75 $\mathrm{g}$ oral glucose tolerance test in women with gestational diabetes. J Obstet Gynaecol Can 28: 690-694

2. Nusrat-Sultana, Hasanat MA, Sharmin-Jahan, Mashfiqul-Hasan, SandeshPanthi, et al. Alarming frequency of Gestational Diabetes Mellitus (GDM) attending a tertiary care hospital in Bangladesh. Submitted to JCD (unpublished)

3. Inturrisi M, Lintner NC, Sorem KA (2011) Diagnosis and Treatment of Hyperglycaemia in Pregnancy. Endocrine Disorder During Pregnancy 40: 703724

4. Tobias DK, Hu FB, Forman JP, Chavarro J, Zhang C (2011) Increased risk of hypertension after gestational diabetes mellitus: findings from a large prospective cohort study. Diabetes Care 34: 1582-1584.

5. Hernandez TL, Friedman JE, Van Pelt RE, Barbour LA (2011) Patterns of glycemia in normal pregnancy: should the current therapeutic targets be challenged? Diabetes Care 34: 1660-1668.

6. Sivaraman SC, Vinnamala S, Jenkins D (2013) Gestational diabetes and future risk of diabetes. J Clin Med Res 5: 92-96.

7. Metzger BE, Buchanan TA, Coustan DR, de Leiva A, Dunger DB, et al. (2007) Summary and recommendations of the Fifth International Workshop-
Conference on Gestational Diabetes Mellitus. Diabetes Care 30 Suppl 2: S251 260

8. Lam KS, Li DF, Lauder IJ, Lee CP, Kung AW, et al. (1991) Prediction of persistent carbohydrate intolerance in patients with gestational diabetes. Diabetes Res Clin Pract 12: 181-186.

9. Dalfra MG, Lapolla A, Mastin M, Gigila G, Dalla BB, et al. (2001) Antepartum and early postpartum predictors of type 2 diabetes development in women with gestational diabetes mellitus. Diabetes Metab 27: 675-80.

10. Tam WH, Ma RC, Yang X, Ko GT, Lao TT, et al. (2013) Prediction of women's long-term cardiometabolic risks using glycemic indices during pregnancy. J Obstet Gynaecol Res 39: 484-491.

11. Lee H, Jang HC, Park HK, Metzger BE, Cho NH (2008) Prevalence of type 2 diabetes among women with a previous history of gestational diabetes mellitus. Diabetes Res Clin Pract 81: 124-129.

12. Kim YL, Cho YK, Park SW, Lee SK, Ahn IS, et al. (2000) Antepartum characteristics predicting persistent post-partum glucose intolerance in the patients with gestational diabetes mellitus. J Korean Diabetes Association 24 46-59.

13. Kitzmiller JL, Kilduff LD, Taslimi MM (2007) Gestational diabetes after deliveryshort-term management and long-term risks. Diabetes Care 30: S225-S235.

14. Johnson K. Predictors of gestational diabetes persistence require followup, International Diabetes Federation (IDF) World Diabetes Congress 2011 Abstract O-0395

15. Kwong S, Mitchell RS, Senior PA, Chik CL (2009) Postpartum diabetes screening: adherence rate and the performance of fasting plasma glucose versus oral glucose tolerance test. Diabetes Care 32: 2242-2244. 